\title{
Direct energy conversion of heat to electricity using AMTEC
}

N. Díez de los Ríos, A. Onea, S. Scherrer, A. Weisenburger and W. Hering IYCE 2015, Pisa

Karlsruhe Institute of Technology (KIT) - Institute for Neutron Physics and Reactor Technology (INR)
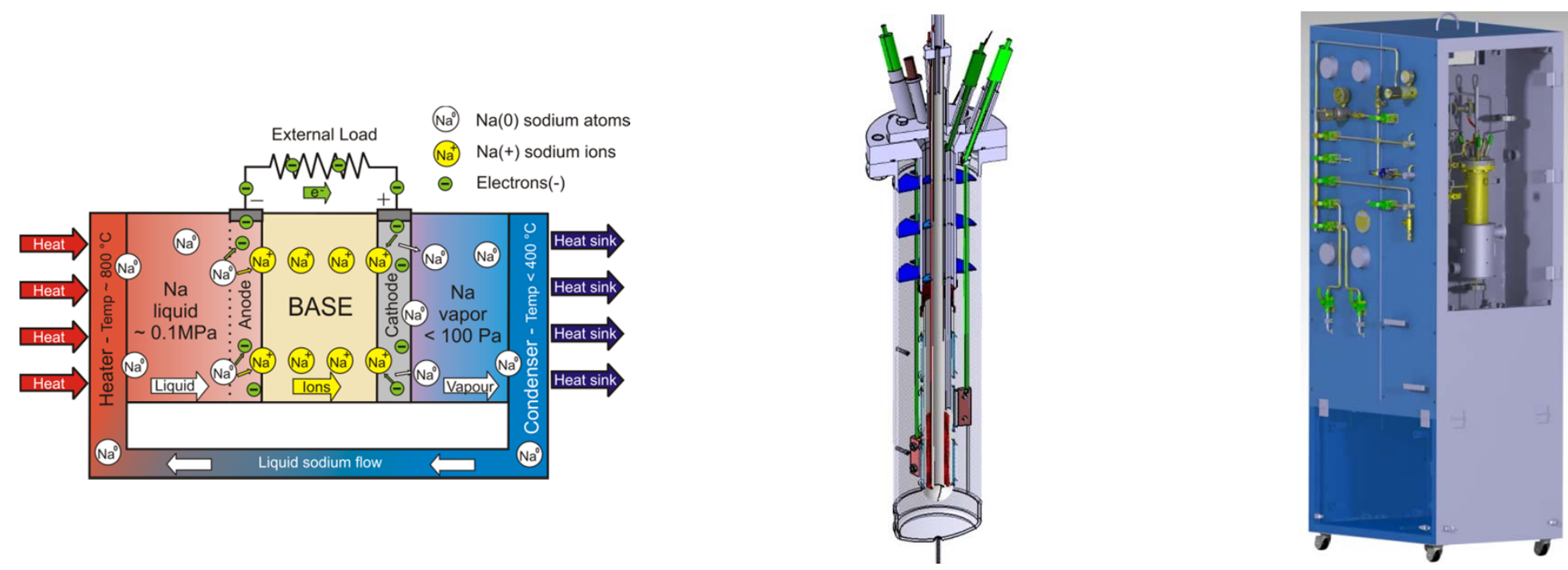


\section{Contents}

1. Introduction: Alkali-Metal Thermal-to-Electric converter (AMTEC)

2. Overview of AMTEC systems

3. AMTEC TEst FAcility (ATEFA) \& Test cell

4. Scoping tests of PEEK in liquid sodium environment

5. Summary and outlook 


\section{AMTEC - direct heat to electricity conversion}

\section{Alkali Metal Thermal to Electric Converter}

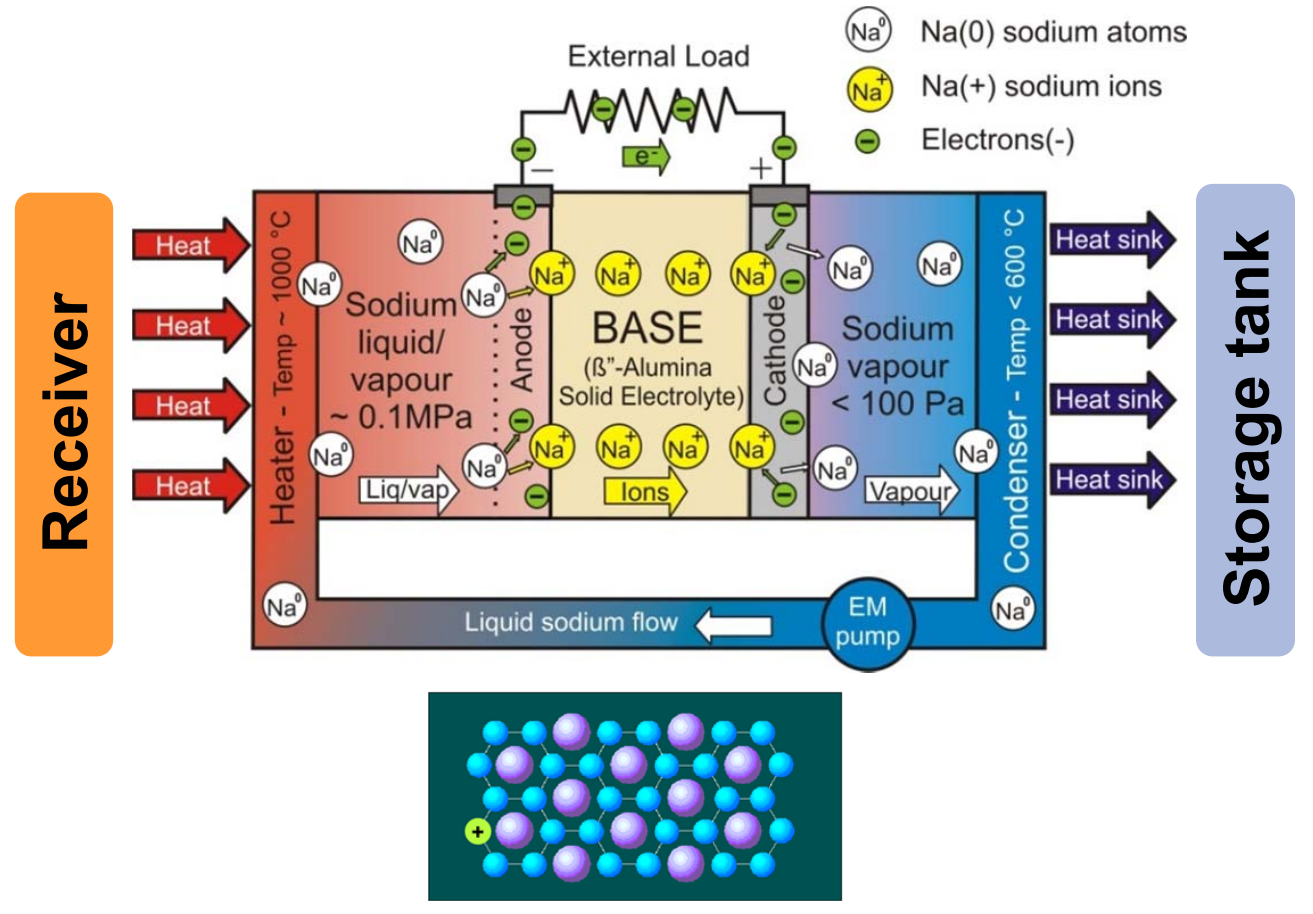

- Key component:

$\beta$ "-Alumina Solid Electrolyte (BASE)

- Key process: Na-ionization

$\Delta \mathrm{P}$ across BASE $\rightarrow \Delta$ (sodium activity)

$$
\mathrm{Na} \rightarrow \mathrm{Na}^{+}+\mathrm{e}^{-}
$$

\section{Main advantages}

- High theoretical efficiency (40\%)

- Flexible regarding the heat source

- Suitable for modular design

- No moving components

\section{Issues}

- Real efficiency of $20 \%$

- Heat losses $(10-15 \%)$

- Electrical losses $(12-15 \%)$

- Power degradation over time

- Degradation of BASE

- Electrode sintering (grain growth) 


\section{Overview of AMTEC systems}

\begin{tabular}{|c|c|c|c|}
\hline Year & Author & $\begin{array}{l}\text { AMTEC cell } \\
\text { characteristics }\end{array}$ & Comments / Electrical properties \\
\hline 1969 & $\begin{array}{l}\text { Kummer \& } \\
\text { Weber }\end{array}$ & $\begin{array}{l}\text { single tube, } \\
\text { liquid anode }\end{array}$ & Developed Sodium Heat Engine (SHE) \\
\hline 1986 & Hunt & $\begin{array}{l}\text { single tube, } \\
\text { liquid anode }\end{array}$ & $\begin{array}{c}\text { Pout }=8 \mathrm{~W}\left(0.4-0.5 \mathrm{~W} / \mathrm{cm}^{2}\right) ; \mathrm{T} \sim 800^{\circ} \mathrm{C} ; \\
\eta=19 \% ; \mathrm{t}_{\mathrm{op}}=1500 \mathrm{~h}\end{array}$ \\
\hline $\begin{array}{c}1989 \\
- \\
1993\end{array}$ & $\begin{array}{l}\text { Heinzel et al. } \\
\text { (KIT - INR) }\end{array}$ & $\begin{array}{l}\text { single tube, } \\
\text { liquid anode }\end{array}$ & $\begin{array}{c}\text { Pout }=112 \mathrm{~W}\left(1.8 \mathrm{~W} / \mathrm{cm}^{2}\right) ; 910^{\circ} \mathrm{C} ; \\
t_{\mathrm{op}}=\text { few hours }\end{array}$ \\
\hline $\begin{array}{c}1992 \\
- \\
1998\end{array}$ & $\begin{array}{l}\text { Air Force } \\
\text { Philips } \\
\text { Laboratory }\end{array}$ & $\begin{array}{l}\text { multitube, } \\
\text { vapor anode }\end{array}$ & $\begin{array}{l}\text { Series connected AMTEC for advanced } \\
\text { radioisotope power system to fulfill the } \\
\text { mission of Pluto Express. } \\
\begin{aligned} \text { Pout }=4.3 \mathrm{~W}\left(0.12 \mathrm{~W} / \mathrm{cm}^{2}\right) ; 750{ }^{\circ} \mathrm{C} ; \\
\eta \sim 12 \% ; \mathrm{t}_{\mathrm{op}}=600 \mathrm{~h}\end{aligned}\end{array}$ \\
\hline 2006 & $\begin{array}{c}\text { Hunt \& } \\
\text { Rasmussen }\end{array}$ & $\begin{array}{c}\text { Small scale, } \\
\text { multitube, } \\
\text { vapor anode }\end{array}$ & $\begin{aligned} \text { Pout }= & 0.5 \mathrm{~W}\left(0.7-0.8 \mathrm{~W} / \mathrm{cm}^{2}\right) ; 740{ }^{\circ} \mathrm{C} ; \\
& \eta \sim 13 \% ; \mathrm{t}_{\mathrm{op}}=5 \text { years }\end{aligned}$ \\
\hline
\end{tabular}

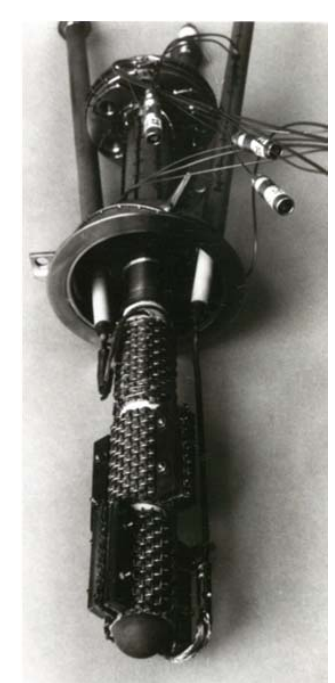

Heinzel et al. 1992

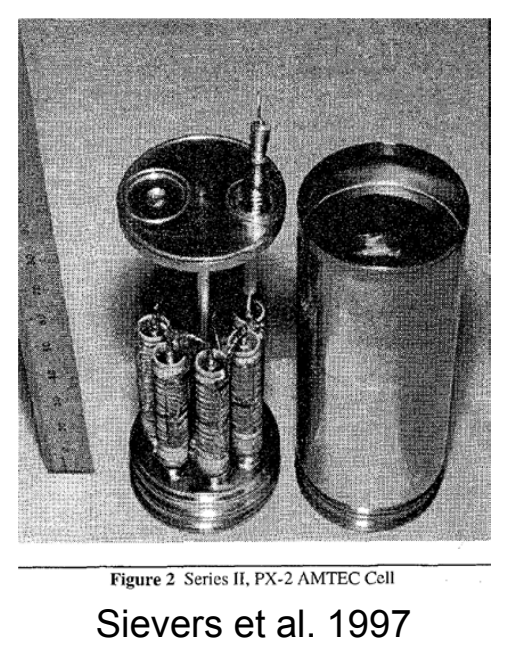




\section{AMTEC TEst FAcility (ATEFA)}

- Research on AMTEC cell

- Test facility consists of Na-side + Ar-side.

- Na-side includes:

- Two storage tanks ( 4,5 I) $\mathrm{T}_{\mathrm{Na}} \sim 150^{\circ} \mathrm{C}$

- AMTEC test cell $\mathrm{T}_{\mathrm{Na}}=600-800^{\circ} \mathrm{C}$

- Air-cooled condenser $\mathrm{T}_{\mathrm{Na}} \sim 300^{\circ} \mathrm{C}$

- Heating trace

- $\mathrm{Vol}_{\mathrm{Na}} \sim 0.3 \mathrm{I}$ (without tanks)

- Ar-side controls Na-flow and pressure in cell and tank

- Max. $p=1.5$ bar

\section{Safe design!}

$\checkmark$ In-housed in a metallic box

$\checkmark$ Possibility to float with $\mathrm{Ar}$

$\checkmark$ Safety equipped lab.

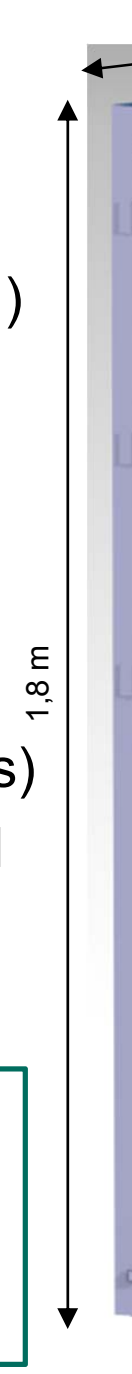

$0,6 \mathrm{~m}$
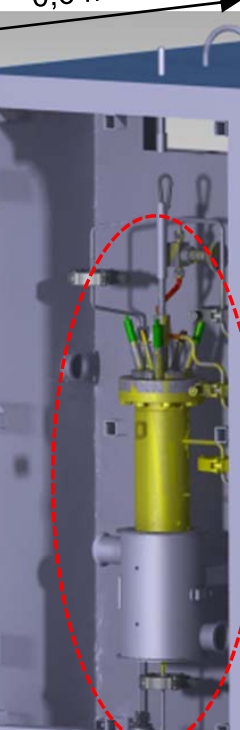

AMTEC

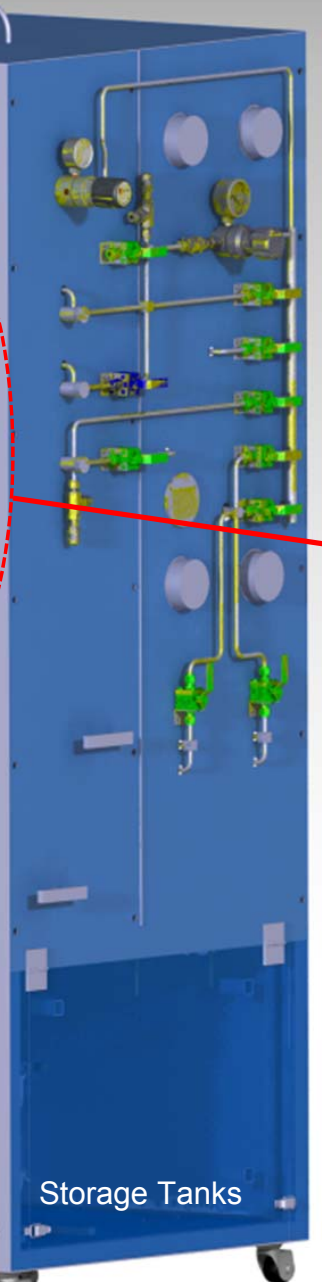
AR-VS-04

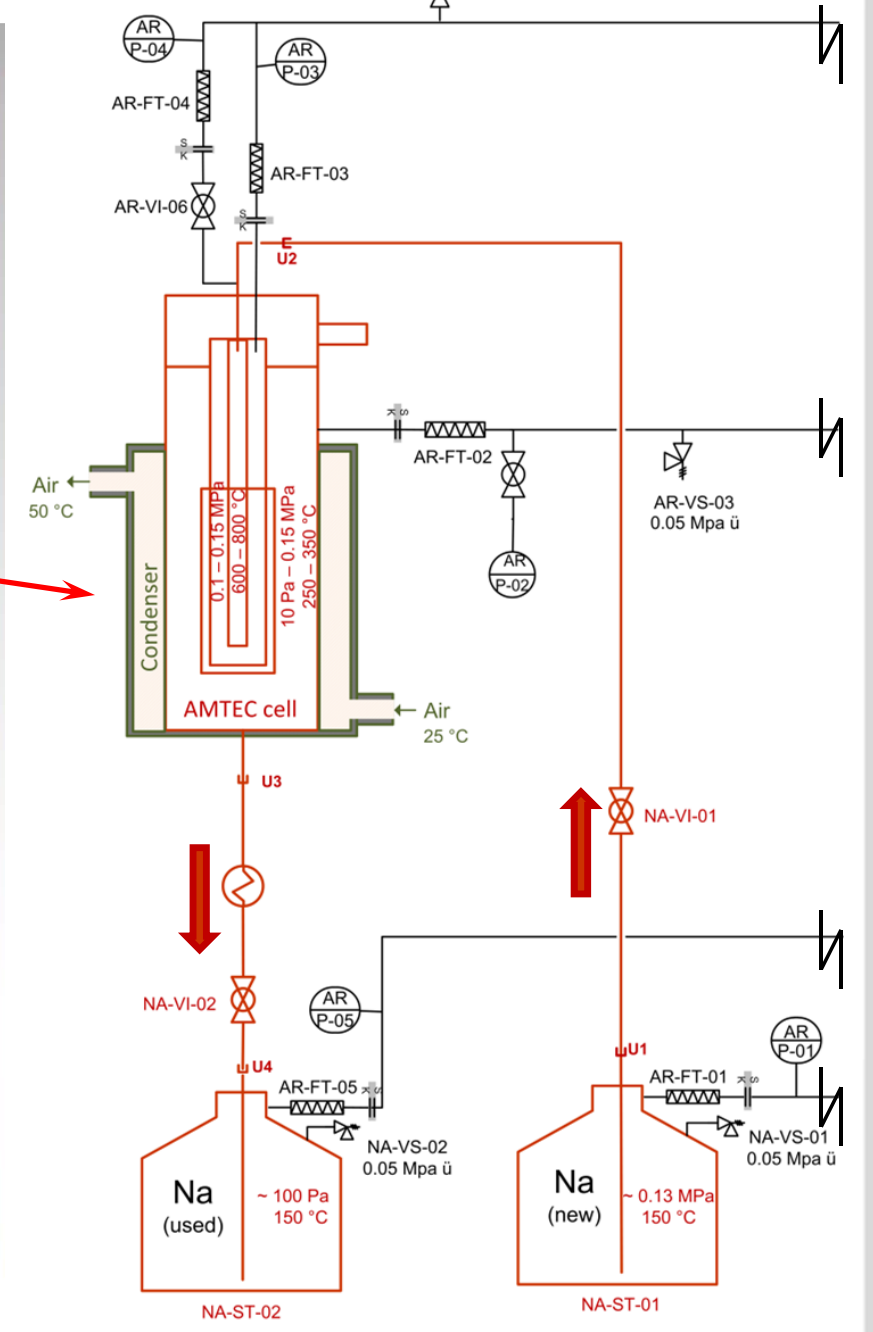




\section{AMTEC test cell}

- Single BASE tube

$(D=3 \mathrm{~cm}, L=22 \mathrm{~cm}$, wall thickness $1 \mathrm{~mm}$ )

- Na-liquid anode

- Sputtered cathodes ( $5 \mu \mathrm{m}$ thickness)

- BASE-Nb-Steel brazing

- 21 thermocouples $\rightarrow \Delta T$ measurement in axial, radial, circumferential direction

- Pressure sensors in Argon atmosphere

- Electronic load $\rightarrow$ IV curve measurements

\section{Tests*}

- BASE (thickness, chemical stability, homogeneity, ionic conductivity)

- Electrode composition (Mo, TiN, TiC)

- Electrode and current collector structure

- Ceramic-metal brazing

*together with other institutes in KIT

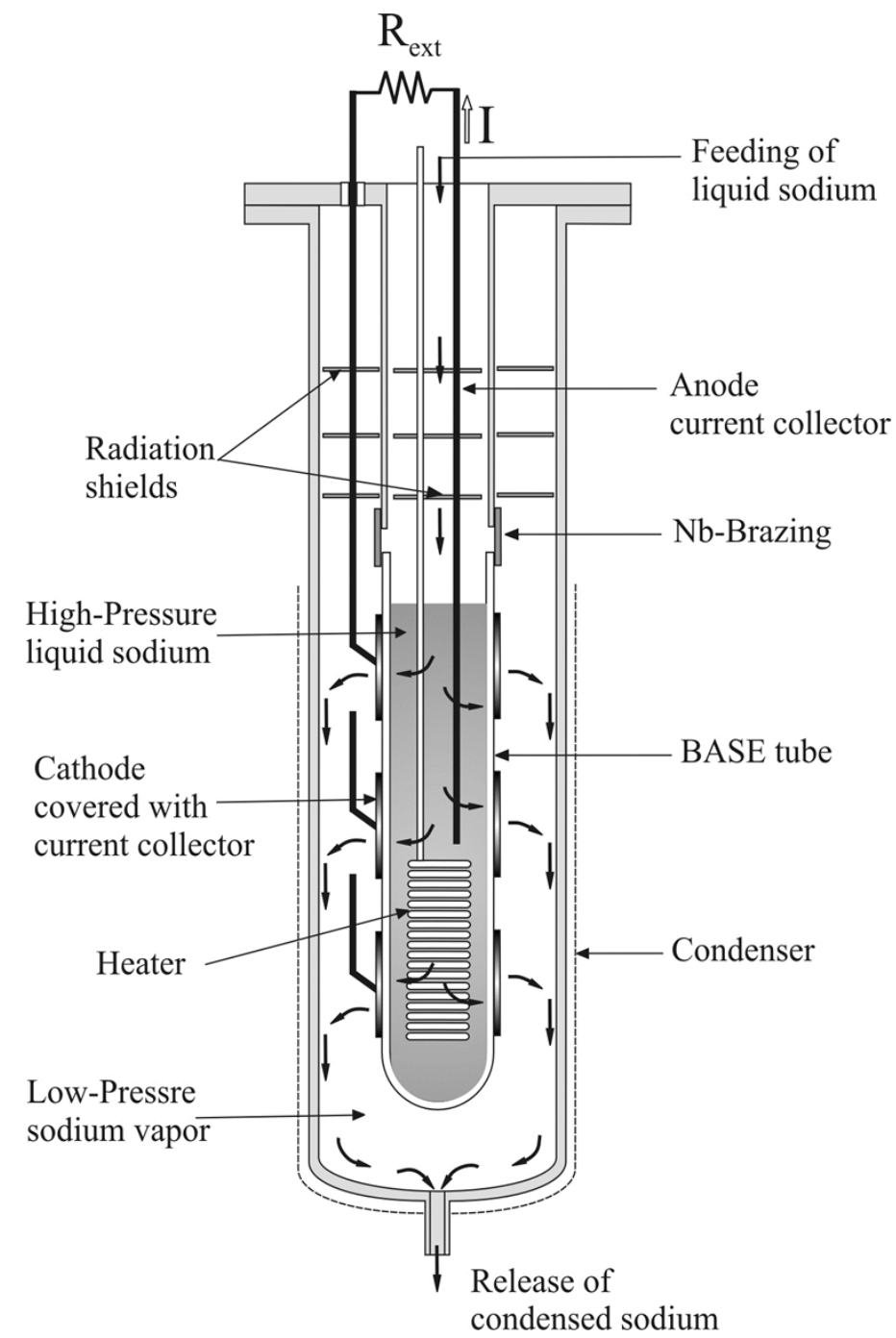




\section{AMTEC test cell}

- Electrode - current collector structure

- Magnetron-Sputtered porous cathode ( $5 \mu \mathrm{m}$ grain size)

- Transport in cathode

- Recombination of $\mathrm{Na}$ ions at triple interface points

- Porosity vs. current density

- Tickness vs. electrical resistance

- Current collector

- Metallic grid, metallic foam, knitted wire...

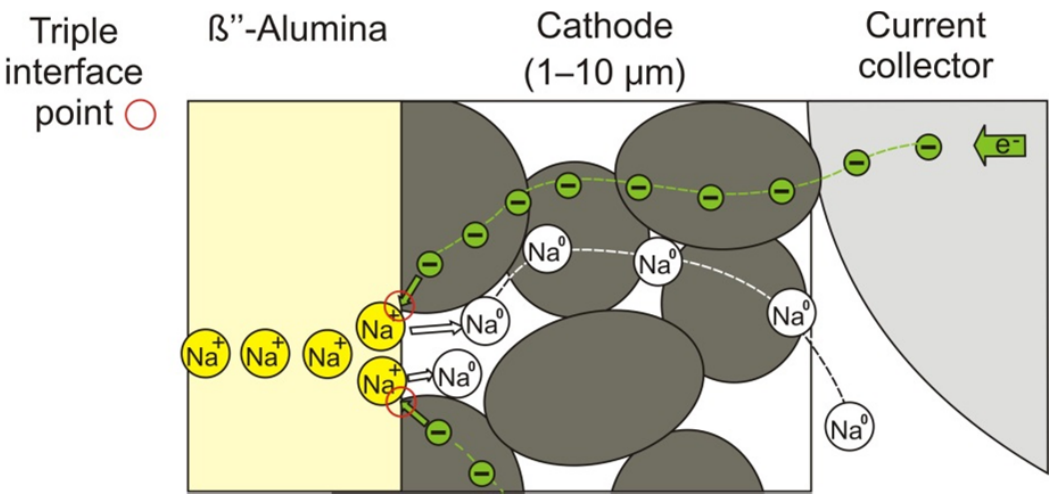

Transport process in the cathode

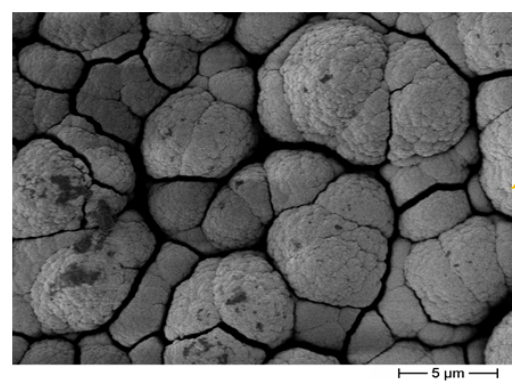

Molybdenum cathode (h $5 \mu \mathrm{m})$
BASE

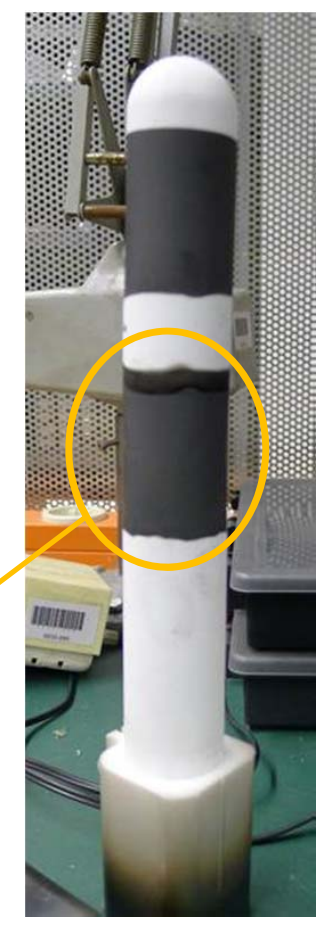

- Sintering of electrode decreases cell performance with time

$\rightarrow$ refractory materials or ceramics (Mo, TiN, TiC) 


\section{Scoping tests of PEEK in liquid sodium}

- Polyether ether ketone (PEEK) is a polymer with excellent mechanical and chemical resistance properties at high temperatures.

- Liquid / vapor sodium is a challenge for sealing materials in valves and instrumentation

- Little information about chemical test of polymers - liquid sodium

- Seven PEEK samples of $\sim 1 \mathrm{~cm}^{2}$ were introduced in a sodium bath at different temperatures inside a glove box with argon atmosphere

- Chemical and mechanical analysis of the samples foreseen

\begin{tabular}{|c|c|c|}
\hline Temp. & Duration & Remark \\
\hline $100{ }^{\circ} \mathrm{C}$ & $15 \mathrm{~min}$. & No changes \\
\hline $120^{\circ} \mathrm{C}$ & $10 \mathrm{~h}$ & No changes \\
\hline $150^{\circ} \mathrm{C}$ & $10 \mathrm{~h}$ & Appearance of black spots \\
\hline $200^{\circ} \mathrm{C}$ & $1 \mathrm{~h}$ & $\begin{array}{c}\text { Sample completely darkened } \\
\text { and apparent changes in } \\
\text { mechanical properties }\end{array}$ \\
\hline
\end{tabular}
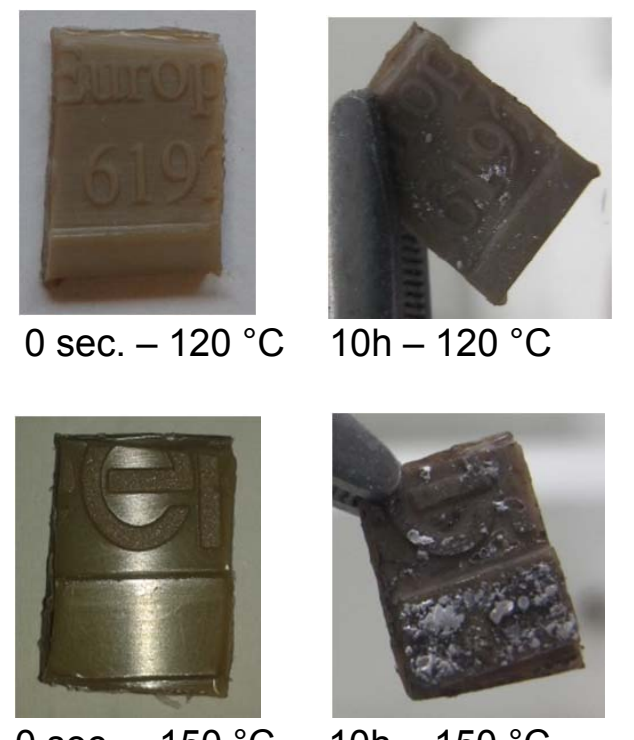

0 sec. $-150^{\circ} \mathrm{C}$

$10 \mathrm{~h}-150^{\circ} \mathrm{C}$

Oberview of the performed PEEK chemical test
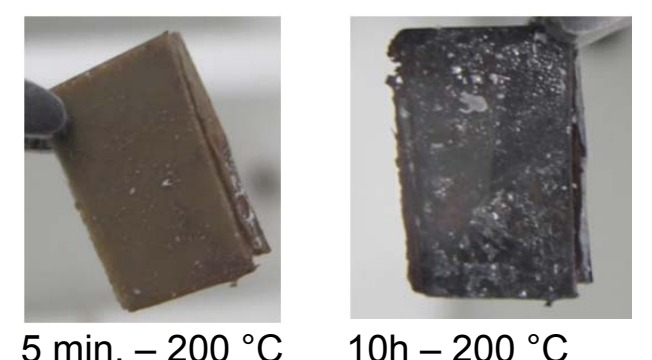

$10 \mathrm{~h}-200^{\circ} \mathrm{C}$

VICTREX-PEEK-450G TM samples before / after chemical test in liquid sodium 


\section{Summary and outlook}

- AMTEC test cell and test facilty have been designed and are under construction

- Tests in sputtering and brazing started

- Scoping test of PEEK in liquid sodium

- Experimental infrastructure (ATEFA facility, AMTEC prototype) to be set into operation by September 2015

- Experimental campaign focused on:

- Open issues (BASE analysis, electrodes)

- Performance evaluation

- Technology improvement

- Further analysis of the PEEK samples

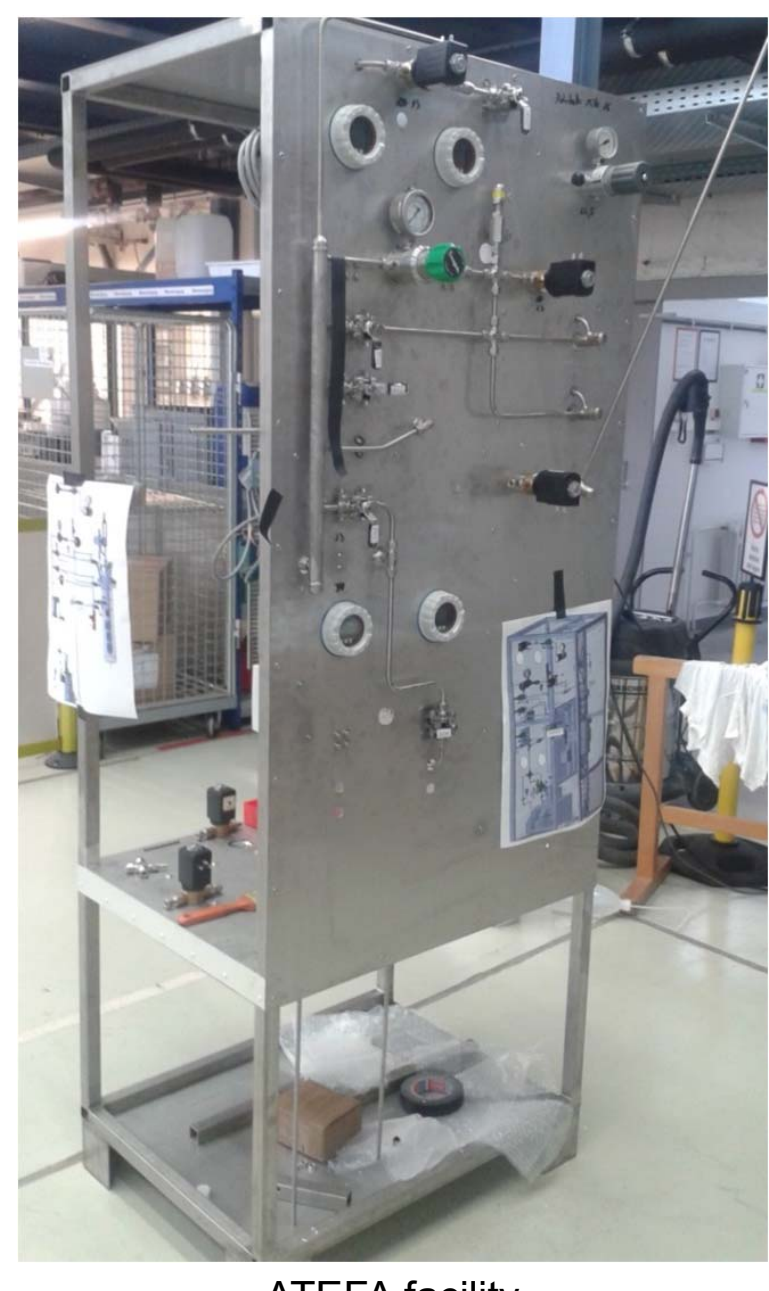

ATEFA facility 


\section{Thank you for your attention}

Contact:

nerea.diez@kit.edu

\section{Funding:}

LIMTECH (Liquid Metal Technology) Alliance $\rightarrow$ HGF Alliance

HEMCP (Helmholtz Energy Materials Characterization Platform) 


\section{Challenges of AMTEC for CSP:}

- Efficiency optimization

- Heat losses $(10-15 \%)$

- Electrical losses (12-15\%)

- Constant performances for long time operation $(\sim 60000 \mathrm{~h})$

- Degradation of BASE (formation of molten dendrites, internal crack formation, contamination through impurities, grain growth)

- Electrode sintering (grain growth)

- Cyclic operation (fatigue)

- Costs: special materials for construction, safety design

- Present technology: $2 \mathrm{MWe}$ with $\sim 30 \mathrm{k}$ BASE elements at $750-800^{\circ} \mathrm{C}$ and $1 \mathrm{~A} / \mathrm{cm}^{2}$
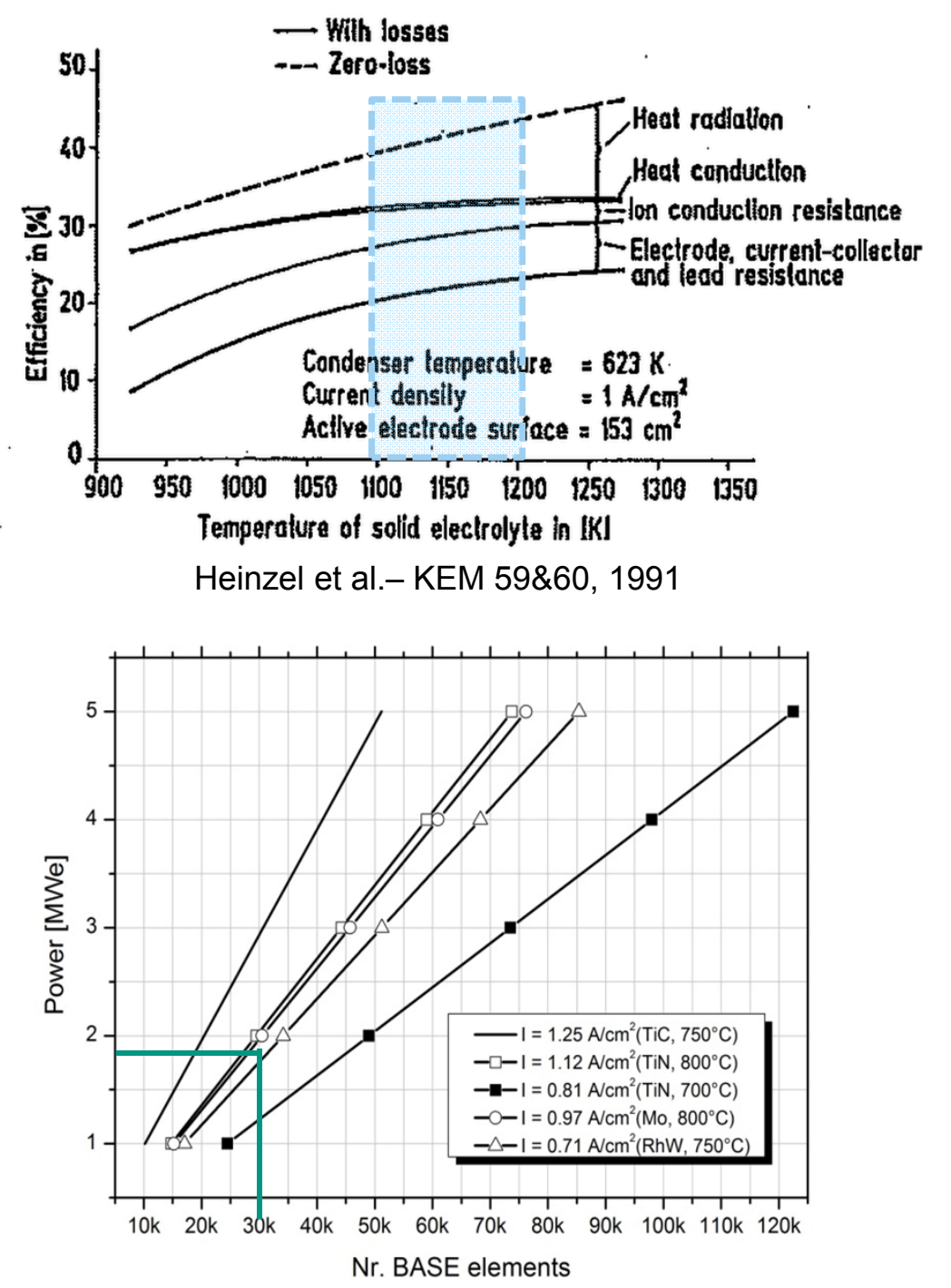

Onea et al. - accepted for publication MHD, 2015 Institute for Neutron Physics and Reactor Technology 


\section{Liquid metals as HTF for CSP}

\begin{tabular}{|c|c|c|c|c|c|}
\hline $\begin{array}{l}\text { Heat transfer } \\
\text { fluid (HTF) }\end{array}$ & $\begin{array}{l}\text { Therminol } \\
\text { VP-1 }\end{array}$ & $\begin{array}{c}\text { Solar Salt } \\
(60 \% \text { NaN03/ } \\
40 \% \text { KNO3) }\end{array}$ & $\begin{array}{l}\text { Liquid metals } \\
\text { (General) }\end{array}$ & $\begin{array}{l}\text { Liquid } \\
\text { Sodium }\end{array}$ & $\begin{array}{c}\text { Steam@ } \\
\text { 10bar }\end{array}$ \\
\hline Stability & $400^{\circ} \mathrm{C}$ & $\sim 585^{\circ} \mathrm{C}$ & $800-2600^{\circ} \mathrm{C}$ & $873^{\circ} \mathrm{C}$ & - \\
\hline $\begin{array}{l}\text { Therm. Cond. } \\
400 / 600{ }^{\circ} \mathrm{C}\end{array}$ & $\begin{array}{l}0.0956 \\
\mathrm{~W} / \mathrm{mK}\end{array}$ & $\geq 0.58 \mathrm{~W} / \mathrm{mK}$ & $>15 \mathrm{~W} / \mathrm{mK}$ & $60 \mathrm{~W} / \mathrm{mK}$ & $\begin{array}{c}0.09 \\
\mathrm{~W} / \mathrm{mK}\end{array}$ \\
\hline Melting Point & $12^{\circ} \mathrm{C}$ & $228^{\circ} \mathrm{C}$ & $<0-300{ }^{\circ} \mathrm{C}$ & $97.7^{\circ} \mathrm{C}$ & - \\
\hline $\begin{array}{c}\text { Density } \\
400 / 600{ }^{\circ} \mathrm{C}\end{array}$ & $696 \mathrm{~kg} / \mathrm{m}^{3}$ & $1867 \mathrm{~kg} / \mathrm{m}^{3}$ & $\begin{array}{l}500 \mathrm{~kg} / \mathrm{m}^{3}- \\
-10000 \mathrm{~kg} / \mathrm{m}^{3}\end{array}$ & $810 \mathrm{~kg} / \mathrm{m}^{3}$ & $2.5 \mathrm{~kg} / \mathrm{m}^{3}$ \\
\hline $\begin{array}{l}\text { Spec. heat } \\
400 / 600^{\circ} \mathrm{C}\end{array}$ & $\begin{array}{c}\sim 2,5 \\
\mathrm{~kJ} / \mathrm{kgK}\end{array}$ & $1,5 \mathrm{~kJ} / \mathrm{kgK}$ & $\begin{array}{c}0,15-4,16 \\
\mathrm{~kJ} / \mathrm{kgK}\end{array}$ & $\begin{array}{c}1,25 \\
\mathrm{~kJ} / \mathrm{kgK}\end{array}$ & $\begin{array}{c}2.2 \\
\mathrm{~kJ} / \mathrm{kgK}\end{array}$ \\
\hline
\end{tabular}

Advantages of $\mathrm{Na}$ :

Thermal stability $870{ }^{\circ} \mathrm{C}$

- High thermal conductivity

- Low melting point

- Low density

Disadvantages of $\mathrm{Na}$

- Reactive with air and water

- Comparison of HTFs *:

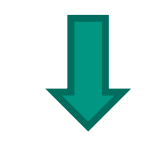

- $\frac{\left(E_{\text {grid }}\right)^{N a}}{\left(E_{\text {grid }}\right)^{\text {Sol Salt }}} \sim 1.06$

- $\frac{\left(E_{\text {grid }}\right)^{N a}}{\left(E_{\text {grid }}\right)^{\text {Steam }}} \sim 1.11$

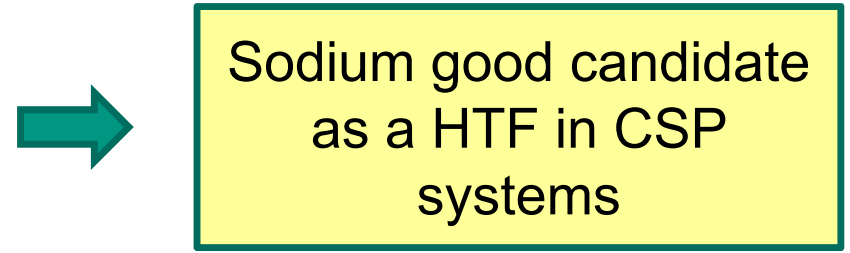

Safety oriented design and maintenance

* Liu et al. SE 101, 220-231, 2014 


\section{Long time perspective Hybrid system CSP + Na + AMTEC}

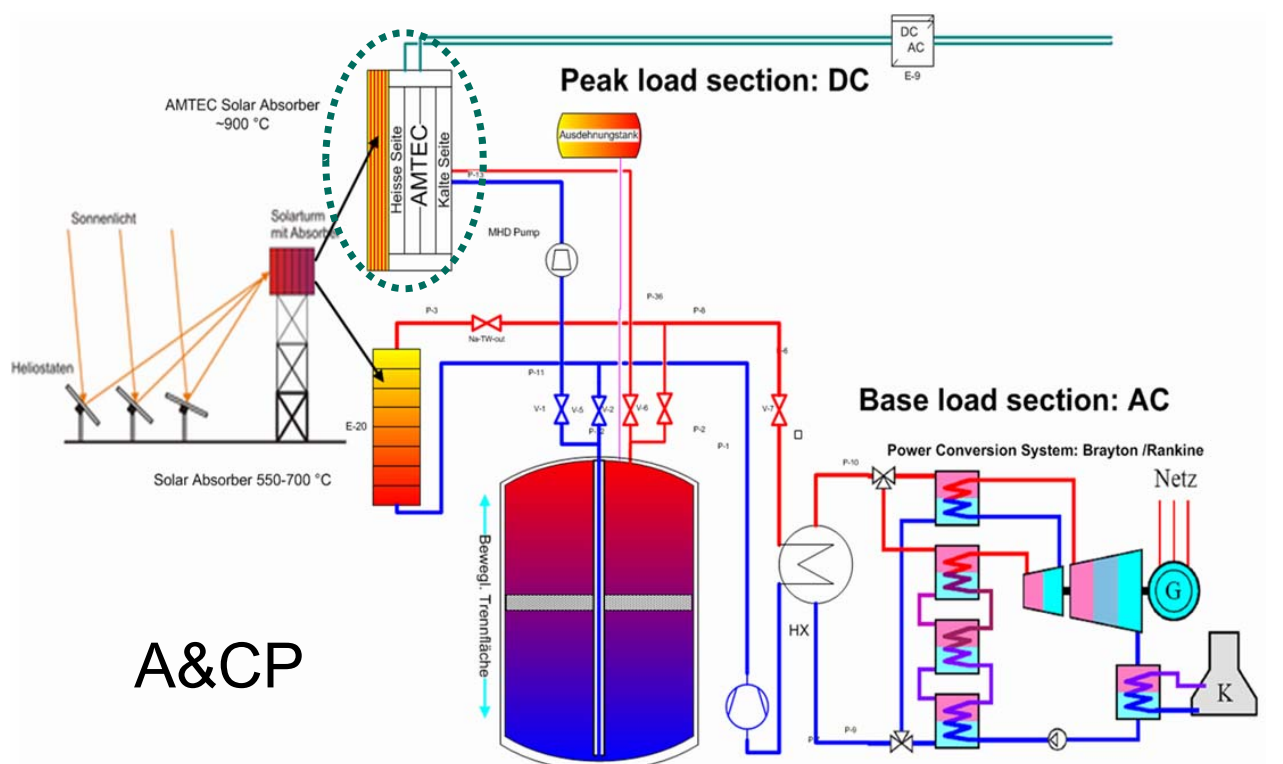

- HTF + storage fluid: $\mathrm{Na}$

- $\mathrm{T}_{\text {receiver }} \sim 550-700^{\circ} \mathrm{C}$

- AMTEC as topping system

- Plant size: $100 \mathrm{MWth}$

- Compensation of fluctuations in source

- AMTEC excess energy reused in TS

- Longer and more flexible operation

- Increase PCS lifetime

- Increase system efficiency* $(\eta \sim 25 \%)$

CSP - AMTEC hybrid cycle (*Hering et al., E2C Maastricht, 2012)

\section{Research in projects LIMTECH, HEMCP}

- AMTEC prototype

- Material characterization in $\mathrm{Na}$

$\rightarrow$ SOdium Loop for Test Materials and Corrosion (SOLTEC)

- High temperature thermal storage device

- A\&CP $\rightarrow$ integration of small prototype in Karlsruhe Sodium Laboratory (KASOLA)

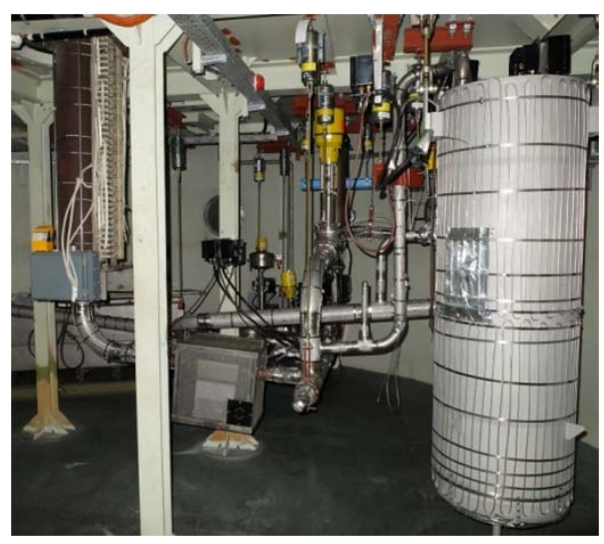

Institute for Neutron Physics and Reactor Technology

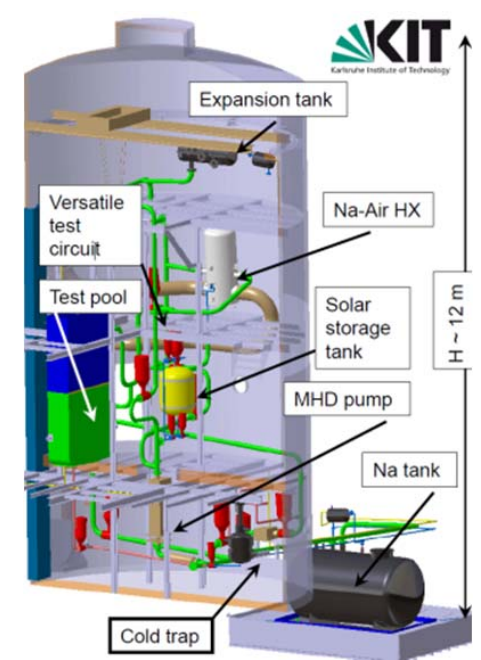

颗NR 


\section{Different direct energy conversion systems}

\begin{tabular}{|c|c|c|l|}
\hline $\begin{array}{c}\text { Energy Conversion } \\
\text { System }\end{array}$ & $\mathrm{T}_{\min } / \mathrm{T}_{\max }\left[{ }^{\circ} \mathrm{C}\right]$ & $\eta[\%]$ & \multicolumn{1}{|c|}{ Comments } \\
\hline $\begin{array}{c}\text { Thermoelectric } \\
\text { converter } \\
\text { (Seebeck effect) }\end{array}$ & $100 / 900$ & $<20$ & $\begin{array}{l}\text { Availability of good } \\
\text { semiconductors that sustain } \\
\text { high temperatures }\end{array}$ \\
\hline $\begin{array}{c}\text { Thermionic } \\
\text { converter }\end{array}$ & $\mathrm{T}_{\max }>1500$ & $<20$ & High operating temperatures \\
\hline Solar cell & $30 / 60$ & $15-30$ & $\begin{array}{l}\text { High costs, "environmentally" } \\
\text { expensive, contain toxic } \\
\text { elements }\end{array}$ \\
\hline Fuel cell & $200 / 1000$ & $35-60$ & $\begin{array}{l}\text { Fuel storage, fuel generation } \\
\text { and lifetime research } \\
\text { needed. High costs. }\end{array}$ \\
\hline AMTEC & $200 / 1000$ & $\begin{array}{c}\sim 20 \\
(40)\end{array}$ & $\begin{array}{l}\text { Lifetime and efficiency under } \\
\text { research }\end{array}$ \\
\hline
\end{tabular}

- All ECS are under development for being competent in the industry

- Issues like lifetime, efficiency, output power and costs need still to be treated

\section{AMTEC - Advantages}

- High theoretical efficiency $(\mathbf{4 0} \%)$

- Flexible regarding the heat source

- Suitable for modular design

- No moving parts

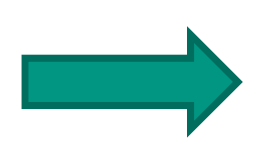

\section{Application for solar energy conversion}

- Silent operation

- Flexible connection (to other heat-to-electric systems) 